\title{
La capacitación como generador de valor empresarial
}

\section{Training as a generator of business value}

\author{
Washington Edy Santillán Marroquín, ${ }^{1}$ \& Héctor Patricio Mera Manotoa. ${ }^{2}$
}

DOI: https://doi.org/10.33262/visionariodigital.v4i3.1269

\begin{abstract}
.
Every time in the organizations the qualification has become a very powerful tool to improve the performance of the workers. In addition, it allows us to systematize processes that the professionalization of the personnel in the company facilitates. It contributes significantly to retain to the human talent as part of its practical emotional wage.

Counting on an efficient qualification, also allows the company to innovate, to develop competitive, corporative and functional strategies, supported in the use of technologies of the information, with an ethical sense and of social responsibility. The qualification must visualize and to be applied in the organizations like a right and an obligation between company and collaborator, the human managers we would have to foment these "good practices" and to create laws that therefore do it. The qualification to the being a planned activity in where all the actors of the company and all the processes of human talent take part to generate a diagnosis landed to the reality of these companies, without a doubt, fortifies the structure of the organization fundamentally and the formation, education and development of the human talent that it toils. Finally, we demonstrated that the qualification influences directly to the development of knowledge, competitions, abilities and skills, fortifying the integral quality of the organization.
\end{abstract}

Keywords: qualification, performance, development, education, competitions, quality

\section{Resumen.}

Cada vez en las organizaciones la capacitación se ha convertido en una herramienta muy potente para mejorar el desempeño de los trabajadores. Además, nos permite sistematizar procesos que facilita la profesionalización del personal en la empresa. Aporta significativamente para retener al talento humano como parte de su salario emocional práctico.

${ }^{1}$ Coordinador Académico del Instituto Superior Tecnológico Bernardo O’Higgins ${ }^{1}$. coordinacion.academica@instituto-ohiggins.com, https://orcid.org/0000-0003-19806832

2 Docente de Marketing del Instituto Superior Tecnológico Bernardo O’Higgins, pato.mera@hotmail.com 
El contar con una capacitación eficiente, también permite a la empresa innovar, desarrollar estrategias competitivas, corporativas y funcionales, apoyado en el uso de tecnologías de la información, con un sentido ético y de responsabilidad social. La capacitación debe visualizarse y aplicarse en las organizaciones como un derecho y una obligación entre empresa y colaboradores, los gestores humanos deberíamos fomentar estas "buenas prácticas" y crear leyes que así lo hagan. La capacitación al ser una actividad planificada en donde intervienen todos los actores de la empresa y todos los procesos de talento humano para generar un diagnóstico aterrizado a la realidad de dichas empresas, sin duda, fortalece la estructura de la organización y fundamentalmente la formación, educación y desarrollo del talento humano que labora. Finalmente, evidenciamos que la capacitación influye directamente al desarrollo de conocimientos, competencias, habilidades y destrezas, fortaleciendo la calidad integral de la organización.

Palabras clave: capacitación, desempeño, desarrollo, educación, competencias, calidad.

\section{Introducción.}

Para una empresa resulta de vital importancia contar entre sus equipos de trabajo con el personal idóneo para el puesto. Conseguirlo es algo que pasa por tres etapas: primero, saber encontrarlo, lo que ya de por sí es un gran reto; en segundo lugar, tener las herramientas y la creatividad para motivarlo, finalmente, y en tercer lugar, saber retenerlo, que en algunos casos puede llegar a ser igual de complejo por la alta competitividad que actualmente tienen las grandes empresas con su talento humano.

Encontrar el trabajador idóneo para un puesto suele ser una tarea de gran responsabilidad, conseguir motivarlo y retenerlo resulta ser muy complejo. La organización debe contar con diversos recursos, todos éstos valiosos, creativos, innovadores, etc., y contar con líderes talentosos a la hora de retener al personal más calificado.

En la actualidad toda empresa requiere un plan de capacitación. Las personas se pueden retirar por voluntad propia, ser despedidas, jubilarse o fallecer dentro de la organización. Las acciones de retención son aquellas prácticas administrativas orientadas a "no dejar ir" a individuos ubicados en cargos claves dentro de la empresa. Lejos de convertirse en acciones que busquen "hacer sentir bien" a los empleados (Harvard, 2003), lo que está en juego es la permanencia de ese factor diferenciador que se expresa en el legado histórico de aprendizaje, materializado en el saber que las personas tienen y que configura lo que algunos denominan el nuevo recurso económico básico, "el conocimiento a través de la capacitación".

$\mathrm{Al}$ respecto no todo trabajador ha llegado a ver la capacitación como un aprendizaje y fortalecimiento de sus competencias frente a su trabajo, más bien el tema de capacitación es tomado por los empleados como algo innecesario y nada productivo, donde se clasifica 
al personal en tres categorías A, B, C; siendo "A" los colaboradores indispensables, los "B" con mayor potencialidad, y los "C" por desarrollar.

No implementar estrategias de capacitación al personal con la intensión de retener y motivar, tiene consecuencias para las organizaciones. "Toda la gestión de la empresa descansa en algún recurso humano encargado de realizar una planeación, diseñar soluciones, toma de decisión, coordinar una ejecución, controlar resultados u operar una acción" (González, 2005, xv). Si son las personas quienes realizan los diversos procesos dentro de las empresas, la capacitación repercute fuertemente en su gestión y en sus resultados.

La retención de talentos es una de las gestiones claves que realiza el área de Talento Humano en su día a día, el saber "retener" al personal calificado es un arte que impacta fuertemente en la productividad y rentabilidad de la empresa.

En procesos de capacitación, la curva de aprendizaje representa el grado de éxito obtenido durante el aprendizaje en el transcurso del tiempo. A medida que una persona aprende más, la productividad o la mejora en la calidad del producto y servicio que realiza serán mayor.

Esta curva de aprendizaje permite visualizar gráficamente las mejoras que se producen en los costos en la medida que los colaboradores ganan experiencia. Al tener la empresa una alta rotación, el aprendizaje será menor y esto repercutirá en un mayor costo y en una baja calidad del producto o servicio que ofrecen al mercado.

Los costos ocultos, entre los cuales, al relacionarlos con la gestión del talento humano, se encuentran el de: captación, contratación e inducción y todos los procesos en que incurren las empresas hasta cuando la nueva persona alcanza el mismo nivel de conocimiento que el trabajador anterior tenía al dejar el cargo; costos indirectos, asociados a la carga de trabajo, a la motivación de los colaboradores y la satisfacción del cliente; además, costos de oportunidad, como la pérdida de conocimientos y de posibilidades por el tiempo requerido para encontrar un reemplazo.

Según Kaplan y Norton (1997), el valor dentro de las organizaciones va de "abajo" hacia "arriba", pasando por la capacitación, aprendizaje continuo, la producción interna, los clientes y las finanzas. Una alta rotación del personal tendrá incidencia en la atención a los clientes ya que una buena relación no se puede desarrollar si una de las partes cambia continuamente, lo cual repercute directa o indirectamente en el consumidor final, impactando en la producción, en el clima laboral y finalmente en las finanzas de la empresa.

La capacitación, educación y desarrollo del talento humano, fortalece una imagen positiva hacia el interior y exterior de la organización. A la vez ésta genera empoderamiento de 
sus colaboradores con la organización, la misma que proyecta una imagen más sólida de su marca en el mercado.

En la era del conocimiento, el capital intelectual es lo que define la ventaja competitiva de una empresa, conjuntamente con un plan de capacitación bien estructurado y focalizado acorde a las necesidades reales del personal.

El conocimiento y las competencias de los trabajadores permiten el surgimiento de nuevas e innovadoras ideas, las cuales se transforman en productos o servicios. Cuando un colaborador valioso sale de la empresa, lo hace con el conocimiento que posee, de tal forma que la empresa no solo pierde al colaborador, sino, todo el saber que él lleva consigo (conocimientos, experiencias y competencias). Si a esto se le agrega la posibilidad que ese saber migre hacia la competencia o se utilice para poner un negocio autónomo, el riesgo para la misma empresa, al “dejarlo ir”, es sumamente alto.

Desde el punto de vista del clima organizacional, la no capacitación del personal tiene también sus consecuencias. La movilidad, sumado a la falta de capacitación del personal genera inestabilidad laboral y un imaginario por parte de los trabajadores de sentirse "utilizados", poco reconocidos y de esta manera se cultiva un clima de desconfianza que repercute negativamente en su quehacer en la organización.

Esta situación trae consecuencias en la imagen que la empresa proyecta de sí misma al exterior, lo cual es poco conveniente en un mercado tan sensible a la percepción de los clientes.

Luego de mencionar algunas razones por las cuales es importante capacitar al personal, se corrobora que, en el ámbito de la gestión del talento humano en las organizaciones, la movilidad laboral y la capacitación se convierten, en un desafío o en una tarea que ha cobrado cada vez más relevancia hasta tornarse en las principales acciones a desarrollar.

Por tal consideración, capacitar y evitar que las personas que ocupan un lugar de vital importancia dentro de la organización se retiren de ella, es hoy en día una prioridad que aporta valor agregado empresarial.

\section{Metodología.}

Un plan de capacitación es un documento elaborado por la dirección de la empresa con la facilitación del área de talento humano, con el fin de asegurar la formación de su personal en el tiempo; el plan integral de capacitación, se inscribe dentro de la estrategia de la empresa y debe contener: el público al que va dirigido, las acciones formativas necesarias, el presupuesto, los resultados esperados la evaluación y el seguimiento de dicha capacitación. (Fernández, Ramírez, \& Díaz, 2008). Al final hay unos pasos para la evaluación de la capacitación entre los cuales se encuentran: la evaluación de necesidades, objetivos de capacitación, contenido del programa, aptitudes-conocimiento, habilidades, 
competencias y evaluación. Lo anterior va dentro de un proceso continuo, a partir de un examen previo al curso y determinar el nivel de conocimientos y necesidad de capacitación; después se implementa la capacitación y un examen posterior para saber que tanto se ha aprendido; finalmente, se realiza un control constante en el cargo asignado y se observa el desempeño que se puede evaluar mediante un formato específico lo que hoy se llama "el seguimiento de la capacitación".

En nuestro país lamentablemente observamos, que la mayoría de empresas privadas que son alrededor del $75 \%$ y que a su vez son de origen familiar, la capacitación ha pasado a un segundo plano y no se le ha dado la importancia vital que esto conlleva. Hay que admitir que los gestores humanos también son co-responsables de esta situación que todavía no han podido "vender" a los líderes la idea de su relevancia y su relación con el talento humano, la productividad y rentabilidad.

Una de las deficiencias que podemos evidenciar en el Ecuador es la falta de leyes estatales focalizadas referentes a la provisión de la capacitación integral a los trabajadores de las empresas, como fuente de atracción, motivación y retención del talento humano valioso.

Sin embargo, en la actualidad las organizaciones estatales tienen ya desarrollado a través de leyes, planes de formación y capacitación para utilizar el potencial de su fuerza laboral y la modernización el estado.

En la organización privada, con el fin de utilizarla como herramienta productiva; ésta todavía no se ha desarrollado eficientemente, ya que a la capacitación la mayoría de los colaboradores no la ven como una inversión si no como un gasto, ya sea de tiempo por parte de los trabajadores y una pérdida económica para el empleador. El trabajador se siente obligado a tomar capacitación no planificada, por lo que no lo hace con una actitud positiva.

El trabajador tiene la responsabilidad de concienciar que a través de la capacitación tiene una valiosa oportunidad para desarrollarse y fortalecer tanto sus conocimientos, habilidades y competencias dentro del ámbito laboral, y a la par, obtendrá una mejora continua en su aspecto personal, familiar y social.

El uso de la capacitación como una herramienta potente debe estar alineada a las políticas de la organización afirmando el compromiso entre sus colaboradores a través de una política interna.

Por lo tanto, esta debería ser vista como un proceso que proporciona conocimientos a sus colaboradores, perfecciona sus habilidades y mejora sus actitudes ante el cliente interno y externo. Uno de los errores más grandes en tiempo de crisis es reducir el presupuesto de la capacitación. La inversión en el capital intelectual es una de las mejores formas para implementar la productividad de la empresa y el desarrollo de la sociedad. 
A través de la capacitación las empresas pueden enfrentarse a los requerimientos actuales y los del futuro, utilizando mejor su potencial humano y así lograr una colaboración más eficiente de su talento (Wurgaft, 1998) Tomando a la Organización Internacional del Trabajo, OIT, es quien define la capacitación como una capacidad efectiva para llevar a cabo exitosamente una actividad laboral plenamente identificada; además, evidencia que vivimos en un mundo cambiante, en donde constantemente hay que formarse, educarse y capacitarse para ser competentes. Por lo tanto, las organizaciones deben poner énfasis en las competencias claves para poder distinguirse de los demás, dichas competencias son un modelo que permite alinear el capital intelectual de una organización con su estrategia de negocios al facilitar simultáneamente el desarrollo profesional de las personas.

La formación y capacitación son una base fundamental para la planificación, desarrollo y evaluación de los sistemas de calidad, en busca de la satisfacción de los clientes y el logro de los resultados de la organización.

La calidad es un concepto que puede tener varios sentidos relacionados, de hecho significa excelencia (Poole, 2010). La actuación de calidad frecuentemente utiliza indicadores internos, de hecho, afecta el proceso de la transformación, la satisfacción del cliente e imagen de la compañía; incluso, la percepción de la posición competitiva con respecto al producto; luego la calidad es un indicador de actuación (Forza, 1995). Según Shridhara (2010, p. 7) "la calidad es la habilidad de un producto o servicio para cumplir con las necesidades de los clientes".

\section{Orientaciones de la capacitación}

Existen dos orientaciones de la capacitación muy influyentes tanto para la empresa como para el trabajador, ambas de gran importancia para aumentar la productividad laboral.

La primera es la capacitación específica, como una herramienta que puede utilizarse para dar soporte a los esfuerzos de construir negocios sustentables al pasar del cumplimiento a adoptar y a integrar factores sociales, ambientales y económicos a las estrategias de los negocios.

La segunda es la capacitación polivalente, que el trabajador recibe en su área de gestión y adquiere conocimientos de toda la operación de la misma; se lleva a cabo bajo la supervisión del coordinador de área y sirve para trabajar no solo en esa organización, sino que también le será útil en el futuro en caso de trabajar en otra empresa o en un puesto diferente.

Entre los beneficios de la capacitación polivalente se tiene el que prepara a la gente para servir a la sociedad durante y después de su retiro; igualmente sirve para validar y fortalecer lo que las personas son y saben, para afianzar su seguridad en el hacer, para promover la paz, la autoestima, la capacidad para vivir, para ser feliz y productivo. 
Es muy importante la capacitación porque integra la organización gracias al diseño de planes como estrategia para el crecimiento y la competitividad; además, pensar en que es una inversión a corto y largo plazo de tipo benéfica para la organización y el colaborador.

Robles (2008, p. 52): "La meta final de toda actividad de formación o capacitación es desarrollar las habilidades del personal, de modo que ejecute las funciones de la organización en forma eficiente, corrigiendo los errores que venían causando problemas y pérdidas económicas"

\section{Beneficios de la capacitación}

La globalización cada vez se convierte en un factor determinante debido a que para poder ser competitivos y permanecer en el mercado, se tienen que desarrollar estrategias que estén acordes y al nivel de las demás empresas. En las organizaciones los recursos son escasos y sumado a una mala asignación e ineficiencia para utilizarlos se generan pérdidas que pueden ser considerables para una empresa; por tal razón, la capacitación se ve como un modelo a seguir para ahorrar recursos, tiempo y dinero, y generar valor agregado al trabajador que no tiene conocimientos previos de cómo realizar una labor y que, luego de tener claro o tener una respuesta a las preguntas de qué, para qué y cómo se debe hacer. Además, genera en el trabajador habilidades para aportar ideas, desarrollar proyectos y tener iniciativa que traen a la organización aportes y/o ideas que realice el trabajador, necesarios y válidos para poder ser competitivos en la industria.

La capacitación es una de las mejores inversiones en el talento humano y una de las principales fuentes de bienestar para el personal y la organización; por eso las empresas y las personas deben ver la capacitación como una inversión que más adelante dará los resultados y metas esperadas.

En cambio, la ausencia de la capacitación trae como consecuencia el incurrir en costos de tipo económico: baja productividad, menor calidad, deficiencia en la atención al cliente, desperdicio en materia prima, infrautilización de máquinas; así como gastos sociales: absentismo, ausentismo, impuntualidad, alto índice de rotación del personal, accidentes, bajas por invalidez e incluso muertes por lo que la imagen y prestigio de la empresa se vería altamente afectada.

\section{Resultados.}

La capacitación bien planificada y focalizada, cuenta con objetivos y resultados muy claros, entre los cuales podemos mencionar:

- Conducir a la empresa a una mayor rentabilidad y a los colaboradores a tener una actitud más positiva.

- Mejorar el conocimiento del puesto en todos los niveles. 
- Elevar la moral y la autoestima de la fuerza laboral.

- Ayudar al personal a identificarse con los objetivos de la empresa.

- Obtener una mejor imagen empresarial. Responsabilidad social corporativa.

- Fomentar la autenticidad, la apertura y la confianza.

- Mejorar la relación líder-subalterno.

- Agilizar la toma de decisiones y la solución de problemas.

- Promover el desarrollo con miras a la promoción.

- Contribuir a la formación de líderes dirigentes.

- Incrementar la productividad y calidad del trabajo.

- Promover la comunicación en toda la organización.

- Reducir la tensión y permitir el manejo de áreas de conflicto.

- Atraer, motivar y retener al talento humano valioso para la organización.

\section{Salario Emocional como Valor Agregado Empresarial}

Es toda retribución no económica que el trabajador puede obtener de la empresa y cuyo objetivo es incentivar de forma positiva la imagen que tiene sobre su ambiente laboral y mejorar su productividad, así como satisfacer las necesidades personales, familiares o profesionales, mejorando su calidad de vida y fomentando un buen clima organizacional.

Este tipo de retribución puede actuar como "factor motivador" de los trabajadores y así mejorar la opinión que tienen de la empresa. Por lo tanto, no se trata de recibir más dinero, sino de recibir otros servicios y compensaciones que el trabajador entiende como beneficios más valiosos que un aumento de sueldo.

Las nuevas generaciones de trabajadores, los millennials por ejemplo, ya no es su más alta prioridad el salario; necesitan, además, ser recompensados con otras retribuciones emocionales que les ayude a conciliar lo profesional con la vida personal y familiar.

Hay seis motivos principales por los que una persona deja su empleo: falta de reconocimiento, que sea regla trabajar fuera del horario establecido ( $\mathrm{u}$ horarios inflexibles), que no se sienta realizado o disfrute su trabajo, mal remunerado, sin posibilidades de crecimiento y que el trabajo no sea flexible. 
Harvard Business Review descubrió que los trabajadores estadounidenses no están realmente comprometidos con su trabajo. Así, los millennials son los menos comprometidos (71\%); con este porcentaje se perfilan como la generación menos implicada con su lugar de trabajo.

El cambio de trabajo en las nuevas generaciones se debe a su perspectiva laboral. La investigación de Harvard muestra que para los millennials es importante estar en una organización donde se les permita crecer integralmente y aprender; que el trabajo que realicen sea interesante y con posibilidad de crecimiento. Mientras para sus padres los ('BabyBoomers') lo importante era contar con un buen jefe y un buen sueldo.

\section{La Capacitación y el Employerbranding}

- El employerbranding es una técnica de marketing dirigida a transmitir dentro y fuera de la organización los valores y características que la definen como empleadora y le permite diferenciarse de otras compañías y obtener una ventaja competitiva dentro de la pugna por contratar a los mejores profesionales.

- Registrará una entrada constante de candidatos interesados en unirse a la plantilla, gracias a su planteamiento a largo plazo.

- La capacitación, el salario emocional y el employerbranding mejoran la fidelización de los trabajadores, reduciendo el absentismo y la rotación de personal.

- Reforzará el sentimiento de pertenencia del capital humano y, con ello, la motivación e implicación de los trabajadores actuales.

- Permitirá a la organización diferenciarse de la competencia a partir de una propuesta de valor añadido.

- Impulsará su imagen de marca general en la sociedad.

- En definitiva, el employerbranding redundará en una notable mejora de los resultados empresariales.

- De esta forma la organización impulsara su imagen de marca en la sociedad, como en su cliente interno.

\section{La capacitación, base del desarrollo económico}

La capacitación de los recursos laborales incrementa la productividad nacional y crea un mercado más competitivo y atractivo para la inversión extranjera. 
La capacitación efectiva de un grupo laboral, mejora la posición de empresas y mercados hacia una posición de competitividad sobre otros mercados que no sean tan efectivos en capacitar a sus recursos laborales.

La capacitación, además de traer beneficios organizacionales y tener un impacto en la competitividad de la industria, es importante para el desarrollo regional; a partir del desarrollo industrial, económico y social basado en el crecimiento que se tiene a nivel industrial se determina el aumento o no en la economía.

De esta manera, la capacitación genera en los colaboradores un conocimiento que es retribuido en la empresa con una mejor eficiencia y en la parte regional con una población más objetiva y mejor calificada, que trae un beneficio en el desarrollo de un país. Esta capacitación tiene que cumplir al final con los objetivos de promover el desarrollo personal y profesional de los trabajadores, mejorar la competitividad de las empresas, adaptar los recursos a las innovaciones tecnológicas y además de eso propiciar el desarrollo de nuevas actividades económicas (Wurgaft, 1998).

\section{Formación y Entrenamiento}

La capacitación como formación es la base de comprensión y habilidades que ayudan a la organización para alcanzar sus objetivos. La capacitación representa uno de los mejores vehículos de desarrollo en cualquier empresa.

El entrenamiento es una clave para sobrevivir, pero debe ser efectivo, trayendo un cambio organizacional positivo. Una organización debe tener clara la relación entre la inversión en el entrenamiento en su puesto de trabajo y el logro de los objetivos organizacionales. Además, se debe desarrollar políticas de capacitación para ampliar un ambiente de aprendizaje junto con una cultura participativa. Es importante que los miembros de la organización desarrollen habilidades de comunicación y comprensión en los diferentes procesos.

La formación es un medio de comunicación, que dota a las personas de habilidades y herramientas para mejorar los procesos; además, ayuda a las personas a crear actitudes hacia la calidad y el servicio.

\section{Conclusiones:}

- La capacitación es un proceso estratégico que deben gestionar de manera sistemática y continua en las organizaciones de hoy, con el fin de que las personas creen los conocimientos y aptitudes necesarias para el desarrollo y sostenimiento de las empresas, en el sentido de mayor productividad y competitividad, por aumento de aceptación de los clientes externos; y a la vez que se logre satisfacer las necesidades de los clientes internos. 
- Lo anterior conlleva a que la organización tenga que diseñar políticas en donde se encaminen actividades y recursos para la formación del personal. Los procesos de formación ayudan a mejorar las habilidades y conocimientos de los trabajadores en las organizaciones de hoy en día en búsqueda de mayor eficiencia, calidad, además del servicio al cliente, dadas las diferentes exigencias y niveles de fiabilidad y calidad.

- En cuanto a los sistemas de calidad, la capacitación permite un buen desempeño y eficiencia en las organizaciones productivas, debido a que se alcanza el desarrollo de la planeación y control de todas las fases de mejora continua, que se hace por medio del enfoque de las habilidades que se requieren en un cargo determinado.

- La empresa moderna tiene el gran reto de implementar planes de capacitación planificados y focalizados acorde a las verdaderas necesidades del personal como parte de su responsabilidad social, el mismo que conlleve a generar un valor empresarial, utilizando el potencial humano y logrando finalmente de ellos, personas eficientes y eficaces.

\section{Agradecimiento:}

- Quisiéramos expresar nuestro profundo agradecimiento a los Alumnos del 3er nivel de la Tecnología de Talento Humano del Instituto Superior Tecnológico Bernardo O'Higgins, por su relevante aporte en la realización de este artículo que fue considerado como proyecto final del módulo de Capacitación de su carrera.

\section{Referencias bibliográficas:}

Auger, A. E. (2006). La capacitación, una alternativa real para los jóvenes.

Cabrera, R. J. A. (2009). ¿por qué es importante la capacitación?

Chiavenato, I. (2000). Administración de Recursos Humanos. Bogotá: Quinta edición. Mc Graw Hill. 108-285

Chiavenato, I. (2011). Planeación estratégica. Fundamentos y aplicaciones. México: Segunda edición. Mc Graw Hill. 72-80

Fernández, C., Ramírez, M., \& Díaz V. (2008). La sinergia y los efectos ignorados de la capacitación: el impacto sistémico en los resultados de las organizaciones turísticas. Retos Turísticos,

Forza, C. (1995). Quality information systems and quality management: a reference model and associated measures for empirical research. Industrial Management y Data Systems, 95 (2), 6-14. doi: 10.1108/02635579510082502

González, M., \& Olivares, S. (2014). Planeación e integración de los recursos humanos: Capital humano (2a. ed.). 
Hernández, C. E. E., \& Molina, A. A. T. (2004). Una alternativa para la evaluación del impacto de la capacitación en las organizaciones empresariales. folletos gerenciales, 12(5) : 16-25, 2008.

Orozco, F. A. (2017). El impacto de la capacitación.

Poole, B. (2010). Quality, semantics and the two cultures. Quality Assurance in Education, 18 (1), 6-18. doi: 10.1108/09684881011015963

Proaño, V. C. A. (2009). Propuesta de un plan de mejoramiento del sistema de capacitación en petroindustrial a ejecutarse en la matriz ubicada en la ciudad de Quito.

Revista Líderes. (2017). El salario emocional tiene más importancia https://www.revistalideres.ec/lideres/salario-emocional-importancia productividad.html

Robles, N. (2008). Metodología para la evaluación de la eficacia y la eficiencia en la capacitación del recurso humano. Revista Tecnología en Marcha, 21 (4), 51-59. Recuperado de: https://dialnet.unirioja. es/descarga/articulo/4835616.pdf

Rodríguez, E. M., \& Ramírez-Buendía, P. (1991). Administración de la capacitación.

Santillán,W.(2017). Nuevas prácticas para mejorar el clima laboral. Quito: Don Bosco.

Werther, W., Keith, D. (2000). Administración de Personal y Recursos Humanos. México: Quinta edición. Mc Graw Hill. 48-225

Wurgaft, J. (1998). La capacitación laboral en los países andinos. Lima, Perú: Organización Internacional del Trabajo. 
PARA CITAR EL ARTÍCULO INDEXADO.

Santillán Marroquín, W. E., \& Mera Manotoa, H. P. (2020). La capacitación como generador de valor empresarial. Visionario Digital, 4(3), 6-18. https://doi.org/10.33262/visionariodigital.v4i3.1269

\section{\Ciencia}

El artículo que se publica es de exclusiva responsabilidad de los autores y no necesariamente reflejan el pensamiento de la Revista Visionario Digital.

El artículo queda en propiedad de la revista y, por tanto, su publicación parcial y/o total en otro medio tiene que ser autorizado por el director de la Revista Visionario Digital.
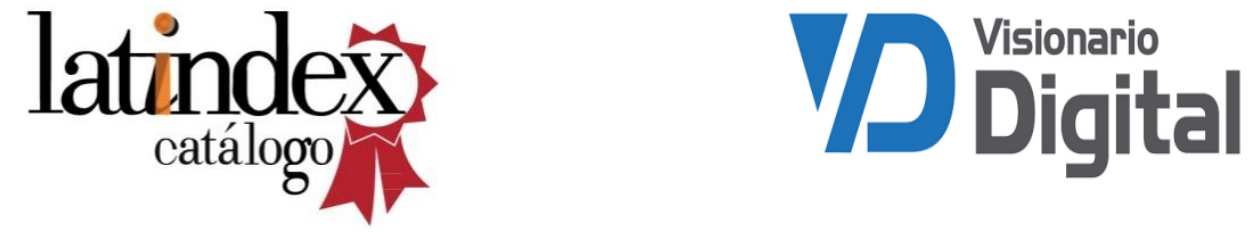